\title{
Nb-Based Nanoscale Superconducting Quantum Interference Devices Tuned by Electroannealing
}

\author{
Simon Collienne $\odot,{ }^{1,}{ }^{*}$ Bart Raes $\odot,{ }^{2}$ Wout Keijers $\odot,{ }^{2}$ Julian Linek, ${ }^{3}$ Dieter Koelle $\odot,{ }^{3}$ \\ Reinhold Kleiner, ${ }^{3}$ Roman B.G. Kramer, ${ }^{4}$ Joris Van de Vondel $\odot,{ }^{2}$ and Alejandro V. Silhanek ${ }^{1, \dagger}$ \\ ${ }^{1}$ Experimental Physics of Nanostructured Materials, Q-MAT, CESAM, Université de Liège, Sart Tilman B-4000, \\ Belgium \\ ${ }^{2}$ Quantum Solid-State Physics, Department of Physics and Astronomy, KU Leuven, Celestijnenlaan 200D, \\ B-3001 Leuven, Belgium \\ ${ }^{3}$ Physikalisches Institut, Center for Quantum Science (CQ) and LISA ${ }^{+}$, Universität Tübingen, Tübingen D-72076, \\ Germany \\ ${ }^{4}$ Université Grenoble Alpes, CNRS, Grenoble INP, Institut Néel, Grenoble 38000, France
}

(Received 26 October 2020; accepted 21 January 2021; published 5 March 2021; corrected 2 July 2021)

In this work, we show that targeted and controlled modifications of the Josephson-junction properties of a bridge-type $\mathrm{Nb}$ nanoSQUID can be achieved by an electroannealing process allowing us to tune and tailor the response of a single device. The electroannealing consists in substantial Joule heating produced by large current densities followed by a rapid temperature quench. We report on a highly nontrivial evolution of the material properties when performing subsequent electroannealing steps. As the current density is increased, an initial stage characterized by a modest improvement of the superconducting critical temperature and normal-state conductivity of the bridges, is observed. This is followed by a rapid deterioration of the junction properties, i.e., decrease of critical temperature and conductivity. Strikingly, further electroannealing leads to a noteworthy recovery before irreversible damage is produced. Within the electroannealing regime where this remarkable resurrection of the superconducting properties are observed, the nanoSQUID can be operated in nonhysteretic mode in the whole temperature range and without compromising the critical temperature of the device. The proposed postprocessing is particularly appealing in view of its simplicity and robustness.

DOI: 10.1103/PhysRevApplied.15.034016

\section{INTRODUCTION}

A dc superconducting quantum interference device (SQUID) is composed of a superconducting loop interrupted by two Josephson junctions. The critical current of the device exhibits an oscillatory response as a function of magnetic field with a periodicity determined by the quantization of the magnetic flux threading the loop. The macroscopic size of a SQUID (usually combined with a suitable input circuit) enables one to realize sensitive magnetic field sensors [1], which are used in a large variety of applications [2]. With the advent of nanotechnology their range of application has been broadened [3], while their energy resolution approaches the ultimate quantum limit $[4,5]$. In recent years, the interest in measuring the

\footnotetext{
*scollienne@uliege.be

†asilhanek@uliege.be

Published by the American Physical Society under the terms of the Creative Commons Attribution 4.0 International license. Further distribution of this work must maintain attribution to the author(s) and the published article's title, journal citation, and DOI.
}

magnetic response of nanoscale systems has launched a number of initiatives to miniaturize these devices $[3,6]$. In this endeavor, conventional Josephson tunnel junctions with insulating barriers are less attractive since their low critical current density prevents them from achieving the optimum performance. With the goal to mitigate this drawback, growing attention has been devoted to Dayem-bridge junctions made of a high critical current density material such as $\mathrm{Nb}$.

Although Nb Dayem-bridge junctions can be nanostructured down to few tens of nm, the short coherence length of the material typically leads to a current-phase relation markedly different from the one measured in ideal Josephson junctions $[7,8]$. This effect, in turn, manifests itself in triangular-shaped critical current oscillations as a function of the applied magnetic field [9], which is detrimental to the SQUID performance, as it suppresses the critical current versus magnetic flux modulation depth [10,11]. Furthermore, poor heat evacuation and high critical currents might lead to the development of self-heating hot spots when the device switches from the superconducting to the normal state. The resultant hysteretic current-voltage $V-I$ characteristics prevent the SQUID from being operated in 
the dissipative regime (voltage mode) as phase coherence is often lost once the hot spots are formed.

Nowadays, the attention has shifted towards postfabrication tweaking of nanoscale SQUIDs in order to optimize their operation performance according to the specific needs. External adjustment of the as-fabricated $\mathrm{Nb}$ SQUIDs, addressing the critical current of the weak links, has been demonstrated to be a promising approach to achieve optimum performance. A nonexhaustive list of controllable junctions includes $\mathrm{Nb}$-normal-metal- $\mathrm{Nb}$ Josephson junction with two additional contacts into the normal region of the junction [12], Nb-semiconductor- $\mathrm{Nb}$ junctions modulated by current injection into the twodimensional electron gas [13], hot-phonon controlled junctions [14], and a multiport $\mathrm{Nb}$ weak-link design [15]. The above described approaches, although elegant and competitive, demand complex multistep nanofabrication procedures and they may become impractical for implementation in confined systems such as SQUID-on-tip devices [16].

In this work, we demonstrate that current-induced modifications of the structure of the weak links through an electroannealing (EA) process provides a viable approach to tune the critical current of $\mathrm{Nb}$ nanoSQUIDs and to suppress their hysteretic response without compromising the operational temperature range of the devices. This paper is organized as follows: in Sec. II we briefly summarize the experimental methods. In Sec. III we present and characterize the electric and magnetic response of the pristine $\mathrm{Nb}$ nanoSQUIDs. In particular, we demonstrate that the critical current oscillations as a function of magnetic field are well captured by a model assuming a linear current-phase relation (CPR). This is a useful reference to compare with the electroannealed devices. Section IV is devoted to the investigation of the physical modifications of the bridges caused by the electroannealing process and its influence on the nanoSQUID's response. Although EA has the tendency to worsen the material properties, by further electroannealing we witness an astonishing recovery allowing us to access a regime in which the SQUID can be operated in a nonhysteretic mode. The conclusion and perspectives for further developments are discussed in Sec. V.

\section{METHODS}

\section{A. Controlled EA}

The electroannealing process is achieved by software control with a feedback loop as described in Ref. [17]. It basically consists in the application of a voltage ramp to attain a constant conductance rate of $-2 G_{0} / s$, where $G_{0}$ is the quantum of conductance, while simultaneously monitoring the consequent resistance change. The software-controlled feedback loop allows reacting upon abrupt resistance jumps and ensures a progressive evolution of the sample's resistance in time. The whole control algorithm is manually stopped either once a certain maximum current, so called the electroannealing current $I_{\mathrm{EA}}$, is achieved, or, for high annealing currents, if a given final resistance is reached. To prevent the sample from electrostatic discharges, a special mounting and connecting protocol as described in Ref. [18] is applied.

\section{B. Cryogenic transport measurements}

The electroannealing is carried out in a cryogenic environment with bath temperature $T_{b} \sim 10 \mathrm{~K}$ above the superconducting critical temperature of $\mathrm{Nb}$. Both, the electrical transport measurements and electromigration process are carried out in a modified commercial Quantum DesignPPMS cryostat. The sample is mounted in a sealed chamber providing a temperature stability better than $1 \mathrm{mK}$ in a He gas atmosphere at 20 mbar.

\section{C. in situ SEM transport measurements}

A specially designed platform permits us to perform EA runs and electrical measurements directly inside a scanning electron microscope. Each EA steps is followed by image acquisition. More details are given in Ref. [19].

\section{PROPERTIES OF THE PRISTINE Nb NANOSQUIDS}

\section{A. Fabrication and electrical characterization}

The investigated samples consist of $t=50$-nm-thick Nb films deposited by electron-beam evaporation onto a $\mathrm{Si}$ substrate terminated with a 100 -nm-thick $\mathrm{SiO}_{2}$ layer. The evaporation is performed under UHV and the $\mathrm{Nb}$ film is subsequently capped with $5 \mathrm{~nm}$ of $\mathrm{Si}$ for protection. Electron-beam lithography is carried out in a Nanobeam nB5 platform with an electron-beam energy of $80 \mathrm{keV}$. Afterwards, an $\mathrm{Al}$ hard mask is fabricated by e-beam evaporation, followed by a lift-off procedure. The $\mathrm{Nb}$, unprotected by the $\mathrm{Al}$ mask, is removed by reactive ion etching with $\mathrm{SF}_{6}$ gas during $10 \mathrm{~s}$. Finally, the $\mathrm{Al}$ is removed by wet etching using the base developer MF-26A. In this study, five samples (labeled S1, S2, .., S5) are characterized, all of them with nominal junction length of $100 \mathrm{~nm}$ and widths between 60 and $80 \mathrm{~nm}$. A scanning electron microscopy (SEM) image of a representative nanoSQUID is shown in Fig. 1(a). The voltage contacts are symmetrically placed at about $1 \mu \mathrm{m}$ away from the constrictions [Fig. 1(a)].

Figure 1(b) shows the superconducting transition of one prototypical device, with resistance $R$, using a dc bias current of $1 \mu \mathrm{A}$. The onset of the superconducting transition defined by the temperature $T$ at which $R(T) / R_{10 \mathrm{~K}}=0.9$, with $R_{10 \mathrm{~K}}$ the normal state resistance of the device at $T=$ $10 \mathrm{~K}$, is located at $T_{c}=8.87 \mathrm{~K}$. A typical low-temperature current-voltage characteristic $V-I$ at zero magnetic field $B=0 \mathrm{mT}$ and $T=1.8 \mathrm{~K}$ is shown in Fig. 1(c). As the 


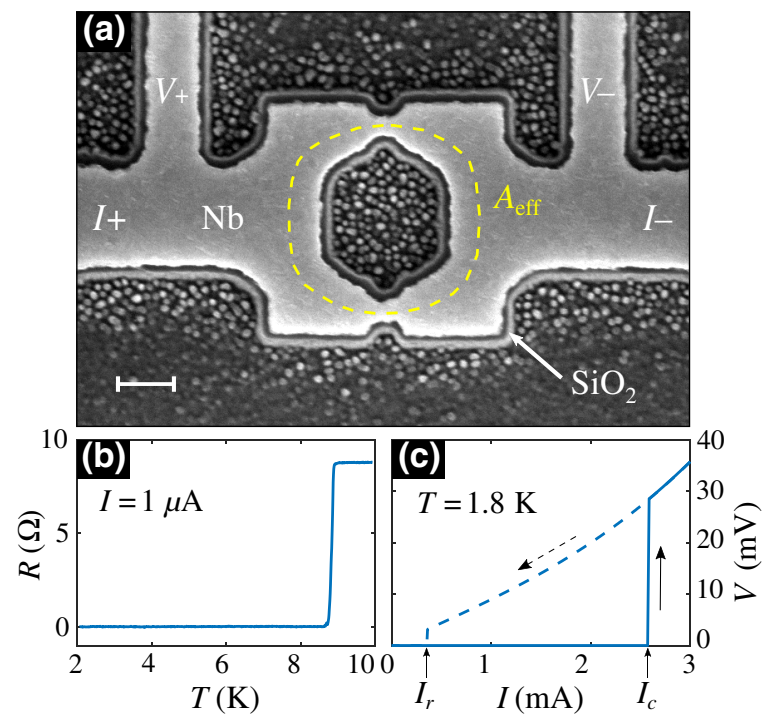

FIG. 1. SEM image of the pristine Nb nanoSQUID S1 (a). The scale bar corresponds to $200 \mathrm{~nm}$. (b) The superconducting transition of the device. (c) The current-voltage characteristic at $1.8 \mathrm{~K}$. The arrows indicate the sweep direction of the bias current. In (a), the yellow dotted line indicates the effective area $A_{\text {eff }}$ of the nanoSQUID.

current bias is gradually increased from zero, the voltage across the device is initially zero and switches abruptly to the resistive state at the critical current $I_{c}$. After a switching event, the device remains in the resistive state due to Joule heating. As the current is subsequently decreased the Joule heating is reduced and the device switches back to the zero-voltage state at the so-called retrapping current $I_{r} \leq I_{c}$.

The normal-state resistance of the nanoSQUIDs exhibits an approximately linear temperature dependence with a thermal coefficient $\alpha=8.5 \times 10^{-3} \mathrm{~K}^{-1}$ between $T=$ $10 \mathrm{~K}$ and $T=300 \mathrm{~K}$ with a residual resistivity ratio $r_{R} \approx 3.2 \pm 0.2$, and a resistivity $\rho(10 \mathrm{~K})=9.7 \mu \Omega \mathrm{cm}$. Using the relation $\rho \ell=3.72 \times 10^{-6} \mu \Omega \mathrm{cm}^{2}$ for $\mathrm{Nb}[20]$, we can estimate a mean free path $\ell=3.9 \pm 0.1 \mathrm{~nm}$. This allows us to calculate the superconducting coherence length at zero temperature using the dirty-limit expression [21] $\xi(0)=0.855 \sqrt{\xi_{0} \ell}=10.5 \pm 0.5 \mathrm{~nm}$, taking $\xi_{0}=39$ $\mathrm{nm}$. In the case of weak-coupling amorphous superconductors in the dirty limit [22], the magnetic penetration depth can be estimated as $\lambda(0)=1.05 \times 10^{-3} \sqrt{\rho / T_{c}} \sim 114 \mathrm{~nm}$.

\section{B. Magnetic field dependence of the critical current}

Figure 2 shows the dependence of the critical current of the nanoSQUID on the applied magnetic field $I_{c}(B)$, at four different temperatures. The value of the critical current is determined from the measured $V-I$ characteristics as a function of the applied magnetic field $B$, using

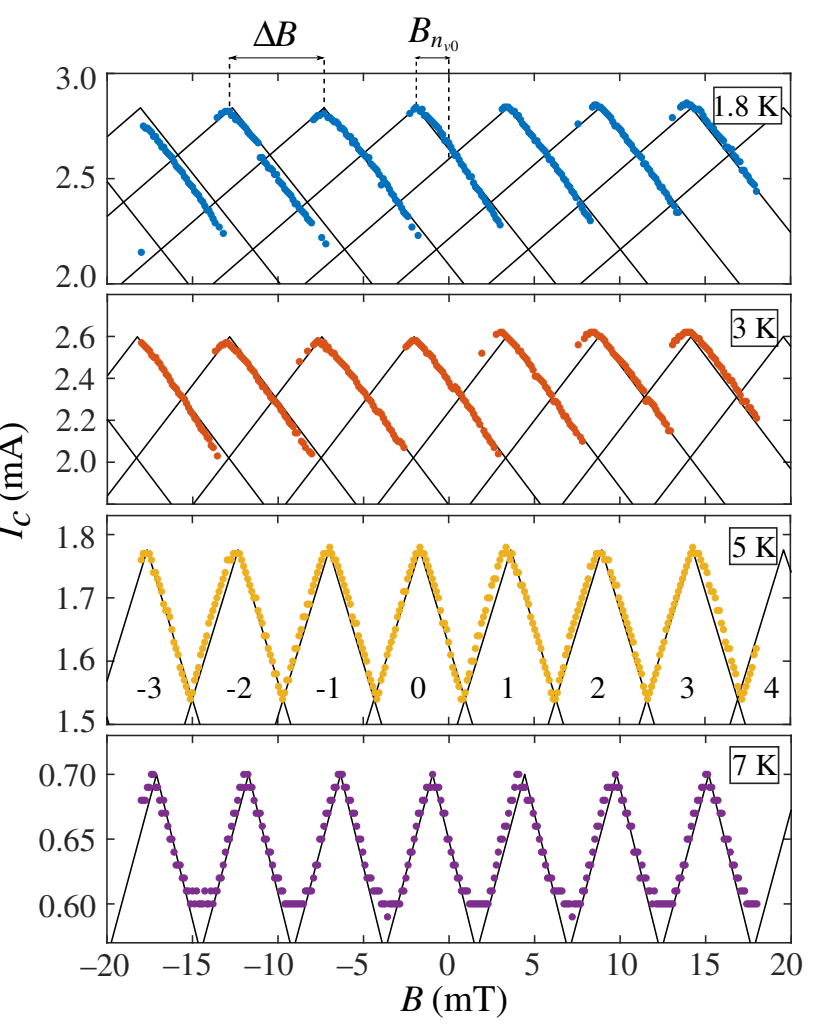

FIG. 2. Critical current of the pristine SQUID S1 as a function of the applied magnetic field for four different temperatures. Black lines correspond to the theoretical prediction assuming a linear current-phase relation according to the model described in Ref. [26]. The numbering from $n_{v}=-3$ to +4 in the panel corresponding to $T=5 \mathrm{~K}$, indicates the vorticity. The fitting parameters obtained from the simulations are listed in Table I.

a $1 \mu \mathrm{V}$ criterion. For the range of explored temperatures, the $I_{c}(B)$ curves show a clear quantum interference effect with a periodicity $\Delta B=5.34 \mathrm{mT}$, from which an effective area $A_{\text {eff }}=0.387 \mu \mathrm{m}^{2}$ roughly twice as big as the actual geometrical area of the inner hole $A_{h}=$ $0.185 \mu \mathrm{m}^{2}$ is obtained. Calculations with the simulation tool 3D-MLSI [23], which solves the London equations for two-dimensional current sheets, results in an effective area $A_{\text {eff }}^{\text {sim }}=0.363 \mu \mathrm{m}^{2}$ for a SQUID with dimensions similar to those of Fig. 1(a) and considering ideal flux focusing [24].

By solving the Ginzburg-Landau (GL) equations, it is shown that for nanobridges longer than approximately $3 \xi(T)$, the CPR becomes double valued and progressively more linear $[10,14]$. For the typical geometrical lengths of the Dayem bridges explored in this work $(d \sim 100 \mathrm{~nm})$, a linear CPR represents a reasonable approximation in the temperature range $T<0.9 T_{c}$ [25]. Although the GL formalism is strictly valid only close to $T_{c}$, an almost linear CPR has been predicted for thin and long wires even at $T=0$ (see Ref. [26] and references therein). The $I_{c}(B)$ curve is composed of approximately linear segments and is multivalued within certain field ranges for $T=1.8 \mathrm{~K}$ and 
$3 \mathrm{~K}$. This linear dependence of the critical current on the magnetic field and the multivaluedness have been observed previously $[10,14]$ and can be accounted for by the model proposed in Ref. [26]. In this model, we assume that each Dayem bridge can be described by a linear CPR:

$$
I_{j}\left(\varphi_{j}\right)=I_{c j} \frac{\varphi_{j}}{\varphi_{c j}},
$$

where the index $j=1$ or 2 indicates the Dayem-bridge number, $I_{j}$ is the corresponding supercurrent, $I_{c j}$ is the critical current, and $\varphi_{j}$ is the phase difference of the macroscopic wave function taken between the end points of the $j$ th Dayem bridge. Further, $\varphi_{c j}$ is the critical phase difference at which the supercurrent reaches its maximum possible value and corresponds to the value above which the Dayem bridge switches to the dissipative state. We additionally assume that the critical current of each Dayem bridge is independent of the magnetic field as expected for the small bridge area and consistent with the experimental observation that the critical current of our nanoSQUIDs oscillates with $B$ around a field-independent mean value. As the order parameter must be single valued, the total accumulated phase around the superconducting loop must be an integer multiple of $2 \pi$ :

$$
\varphi_{1}-\varphi_{2}+2 \pi \frac{B}{\Delta B}=2 \pi n_{v}
$$

where $n_{v}$ is the vorticity (i.e., the winding number of the macroscopic wave function) of the SQUID loop.

The total critical current of the nanoSQUID, $I_{c}(B)$, is determined from Eqs. (1) and (2) as the smallest total applied current $I=I_{1}+I_{2}$ at which the phase difference across either bridge reaches its critical value $\varphi_{j} \geq \varphi_{c j}$. In addition to the approach presented in Ref. [26], we introduce two additional constraints allowing us to reduce the number of fitting parameters to only two $\left(I_{c 1}\right.$ and $\left.\varphi_{c 1}\right)$ instead of four $\left(I_{c j}\right.$ and $\left.\varphi_{c j}, j=1,2\right)$. Firstly, note that the maximum critical current of the nanoSQUID, given by $I_{c}=I_{c 1}+I_{c 2}$, takes place when both bridges reach their corresponding critical currents and critical phases. As we can determine $I_{c}$ from the experimental data, this constraint allows us to eliminate one of the two critical currents as a fitting parameter. Secondly, the magnetic field value corresponding to the maximum of the $n_{v}=0$ branch is given by

$$
B_{n_{v 0}}=\frac{\Delta B}{2 \pi}\left(\varphi_{c 2}-\varphi_{c 1}\right),
$$

(see Fig. 2). Equation (3) allows us to eliminate one of the two critical phases differences as a fitting parameter. Note that Eq. (3) indicates that a shift of the $I_{c}(B)$ curves along the $B$ axis results from an asymmetry in $\varphi_{c j}$ for the two junctions. The results of the fitting procedure are depicted by solid black lines in Fig. 2 and the corresponding fitting parameters are shown in Table I. The multivalued character of the $I_{c}(B)$ curve at low temperatures is properly captured by the model and results from the fact that different values of $n_{v}$ gives rise to different critical current branches [26].

This model further assumes that the response is dominated by the kinetic inductance. A first-order calculation of the geometric self-inductance of the loop gives approximately $0.7 \mathrm{pH}$ [27]. For comparison, we can estimate the contribution of the kinetic inductance to be determined by $L_{K}=\mu_{0} \lambda(0)^{2} \int_{C} d r / A(r) \sim 1.9 \mathrm{pH}$, where $C$ is the curvilinear path with the coordinate $r$ along one arm of the SQUID and $A(r)$ is the position-dependent cross section of the conductor. Independently, $L_{K j}=\varphi_{c j} \Phi_{0} / I_{c j} 2 \pi$, with $\Phi_{0} \approx 2.0678 \mathrm{mT} \mu \mathrm{m}^{2}$ is the magnetic flux quantum, and therefore it can be deduced from the fitting parameters. The obtained values listed in Table I confirm that the kinetic energy indeed dominates the response of the nanoSQUID.

Table I shows that the asymmetry in critical current of the Dayem bridges $\alpha_{I}=\left|I_{c 1}-I_{c 2}\right| /\left(I_{c 1}+I_{c 2}\right)$ is independent of temperature and remains around $\alpha_{I} \sim 0.05$ expected from geometrical inspection. The critical phase differences of both nanobridges exceed the value of $\pi / 2$ expected for tunnel junctions and decrease with increasing temperature. This observation is in agreement with the simulations carried out within the GL formalism by Hasselbach et al. [10]. Furthermore, according to Ref. [26], the effective length $d_{\mathrm{eff}} \sim 4 \xi \varphi_{c} / \pi>d$ for all temperatures. This result suggests an extended phase distribution spreading over a distance larger than the bridge's length [25].

\section{ELECTROANNEALED DEVICES}

Let us now focus on the modification of the material properties at the constrictions by the controlled process of electroannealing. Within this process, a bias voltage across the device is slowly swept up while simultaneously monitoring the decrease of conductivity until reaching a pre-established value. Sudden decreases of conductivity leading to a thermal runaway and eventually sample destruction are avoided by a reactive feedback loop [17]. Unlike electromigration [19], EA is mainly driven (but not only) by the Joule heating produced by high current densities only achievable in high melting-point materials such as $\mathrm{Nb}$. We have recently reported the successful implementation of this approach [28] for producing targeted modifications of the superconducting properties in bow-tie $\mathrm{Nb}$ nanoconstrictions. In this work, this procedure is extended to two parallel constrictions following the protocol described in Ref. [17].

\section{A. Targeted damage at the weak links}

Performing the EA process at room temperature inside the chamber of a scanning electron microscope allows us to monitor in situ the structural changes occurring in 
TABLE I. Fitting parameters for the $I_{c}(B)$ oscillations of the pristine state. The free fitting parameters are the critical current $I_{c 1}$ and phase $\varphi_{c 1}$ of Dayem bridge 1. The values for Dayem bridge 2 are obtained from Eqs. (2) and (3). $\alpha_{I}=\left|I_{c 1}-I_{c 2}\right| /\left(I_{c 1}+I_{c 2}\right)$ indicates the asymmetry in critical currents and $L_{K j}=\varphi_{c j} \Phi_{0} /\left(I_{c j} 2 \pi\right)$.

\begin{tabular}{lccccccc}
\hline \hline$T(\mathrm{~K})$ & $I_{c 1}(\mathrm{~mA})$ & $\varphi_{c 1}(\mathrm{rad})$ & $I_{c 2}(\mathrm{~mA})$ & $\varphi_{c 2}(\mathrm{rad})$ & $\alpha_{I}$ & $L_{K 1}(\mathrm{pH})$ & $L_{K 2}(\mathrm{pH})$ \\
\hline 1.8 & $1.8 \pm 0.5$ & $20 \pm 5$ & $1.1 \pm 0.5$ & $18 \pm 5$ & $0.2 \pm 0.3$ & $3.7 \pm 1.4$ & $5.4 \pm 2.9$ \\
3 & $1.4 \pm 0.2$ & $15 \pm 2$ & $1.2 \pm 0.2$ & $13 \pm 2$ & $0.1 \pm 0.1$ & $3.5 \pm 0.7$ & $3.6 \pm 0.8$ \\
5 & $0.97 \pm 0.08$ & $12 \pm 1$ & $0.81 \pm 0.08$ & $11 \pm 1$ & $0.1 \pm 0.1$ & $4.1 \pm 0.5$ & $4.5 \pm 0.6$ \\
7 & $0.39 \pm 0.03$ & $8.5 \pm 0.8$ & $0.31 \pm 0.03$ & $7.4 \pm 0.8$ & $0.1 \pm 0.1$ & $7.2 \pm 0.9$ & $7.9 \pm 1.1$ \\
\hline \hline
\end{tabular}

the device under thermal stress. The upper left panel of Fig. 3 shows the values of the maximum resistance $\left(R_{\max }\right)$ obtained at a current $I_{\mathrm{EA}}$ and the minimum resistance $\left(R_{\min }\right)$ recorded after the current has been reduced to $1 \mu \mathrm{A}$. For $I_{\mathrm{EA}} \leq 8 \mathrm{~mA}$, the increase of $R_{\max }$ with increasing $I_{\mathrm{EA}}$ results primarily from Joule heating, as confirmed by the fact that no change of $R_{\min }$ is observed in this current range. The threshold beyond which irreversible modifications in the constrictions are induced corresponds to $I_{\mathrm{EA}}=8 \mathrm{~mA}$. For each EA step we acquire SEM images immediately after the device is submitted to thermal stress. A selected set of these images is shown in Figs. 3(a)-3(d). The panel corresponding to the pristine sample, evidences a small asymmetry in the width of both bridges. Panel (a) shows that exceeding the $8-\mathrm{mA}$ threshold current leads to clear material alteration localized at both bridges. These modifications are identified as significant brightness contrast in the SEM signal in comparison with the pristine state. The observed increased modifications on both bridges is directly correlated with an increase of the overall resistance of the device. Panel (b) shows the development of a nanogap in the narrower bridge, which becomes more apparent in panels (c) and (d). Additional data acquired from sample S2 confirm these observations (see Supplemental Material [29]). The fact that the gap is generated in the narrower constriction confirms previous findings [19]

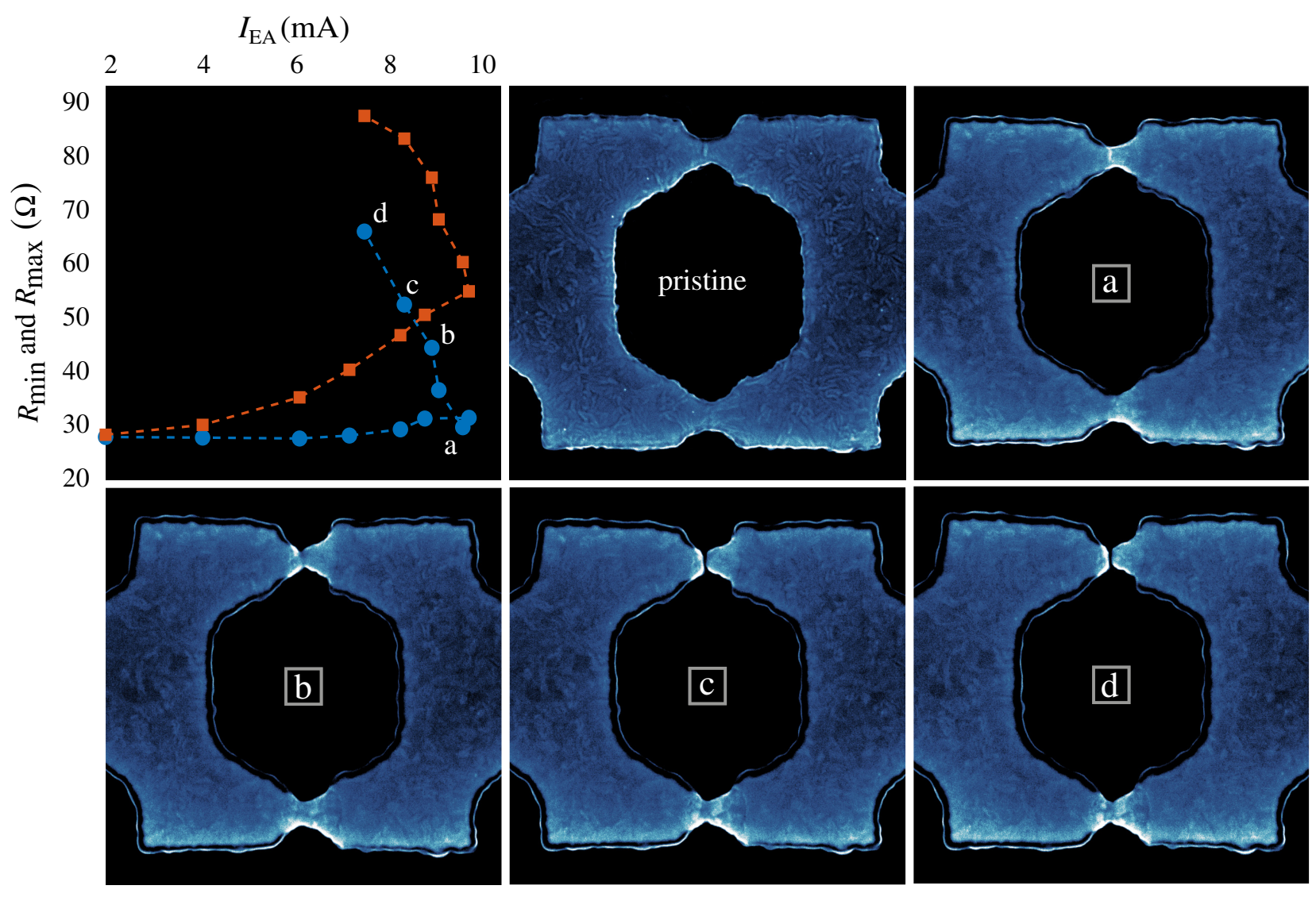

FIG. 3. SEM images obtained during the electroannealing process for sample S3. The upper left panel shows the values of the maximum resistance $\left(R_{\max }\right)$ in red (squares) obtained at a current $I_{\mathrm{EA}}$ and the minimum resistance $\left(R_{\min }\right)$ in blue (dots) obtained after the current has been reduced to $1 \mu \mathrm{A}$. (a)-(d) selected SEM images after the device has been submitted to EA. 


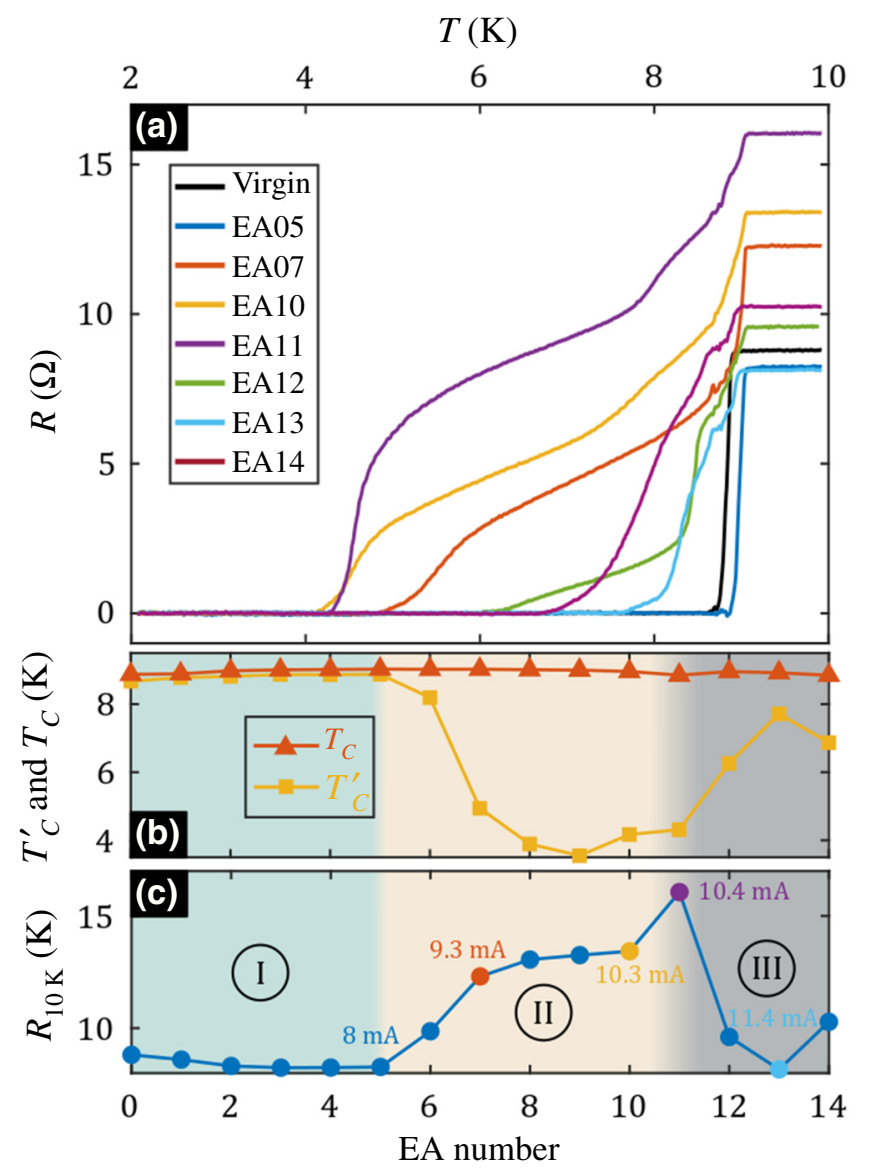

FIG. 4. (a) Selected set of $R(T)$ curves for sample S1 measured with an applied current $I=1 \mu \mathrm{A}$ after subsequent electroannealing processes. (b) Evolution of the critical temperature of the weak link $T_{c}^{\prime}$ and the critical temperature of the arms of the SQUID $T_{c}$. (c) The normal-state resistance $R_{10 \mathrm{~K}}$ as a function of the EA number. $I_{\mathrm{EA}}$ is given for some EA steps with the same color code as in (a). Regions I, II, and III are described in the text.

and indicates that the atomic migration regime driven by high current density has been achieved. Clearly, during the EA process with the scope of tweaking the SQUID properties, the extreme situation of generating a gap in one of the weak links, which would suppress the SQUID oscillations, is to be avoided.

An estimation of the local temperature in the weak links at the onset of electroannealing of $T \sim 400-460$ $\mathrm{K}$ can be obtained from the $R(T)$ response of the sample and COMSOL simulations (see Supplemental Material [29]). Interestingly, this value does not seem to depend on the bath temperature. In other words, it is irrelevant whether the EA is performed under cryogenic conditions or at room temperature. Although $\mathrm{Nb}$ has an oxide coating consisting of amorphous and insulating $\mathrm{Nb}_{2} \mathrm{O}_{5}$, which protects the superconductor against further oxidation from the atmosphere, above $400 \mathrm{~K}$ oxygen dissolves into $\mathrm{Nb}$ and

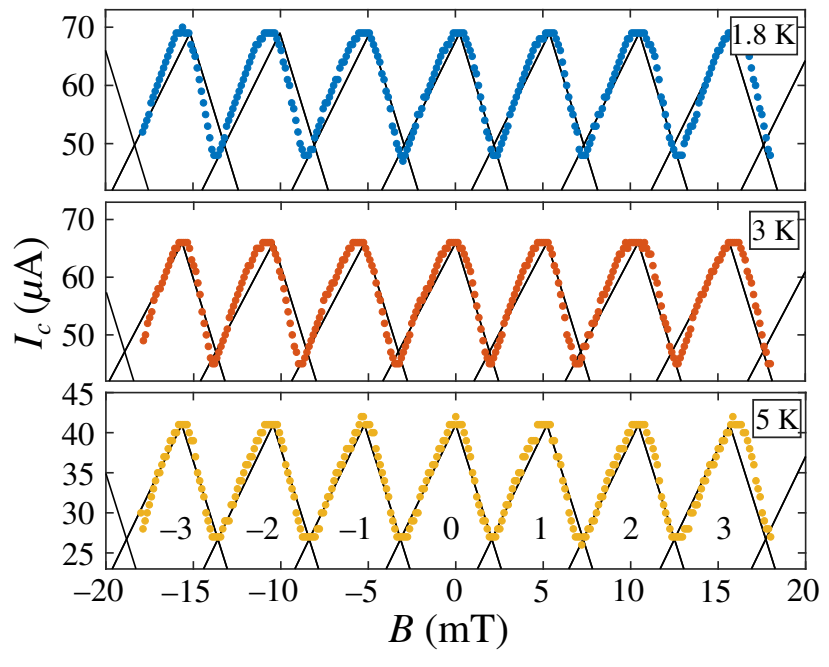

FIG. 5. Critical current of the SQUID as a function of the applied magnetic field for three different temperatures after EA14 (sample S1). The parameters of the simulations are listed in Table II. The numbering from $n_{v}=-3$ to +3 in the panel corresponding to $T=5 \mathrm{~K}$, indicates the vorticity.

deteriorates the superconducting properties [30-32], this can possibly explain the observed material alteration during the EA process. Additional atomic force microscope (AFM) experiments are available for sample S5 in the Supplemental Material [29] and confirm that the EA-induced changes are mostly localized at the two junctions of the SQUID.

\section{B. Characterization of the superconducting properties of the weak links after electroannealing}

Figure 4 shows the typical evolution of the normalstate resistance and the superconducting transition temperature observed after subsequent EA processes carried out at $10 \mathrm{~K}$. For the sake of clarity, a selected set of $R(T)$ curves measured with an applied current $I=1 \mu \mathrm{A}$ are shown in (a). The first observed effect after few initial EA steps $\left(\mathrm{EA} 01 \rightarrow \mathrm{EA} 05, I_{\mathrm{EA}} \leq 8 \mathrm{~mA}\right)$, consists in a modest improvement of the superconducting transition $\delta T_{c} \sim 150 \mathrm{mK}$ accompanied by a simultaneous decrease of the normal-state resistance of the device [see region I in Figs. 4(b) and 4(c)]. Although this effect is relatively weak, it is beyond the experimental error bar and consistently observed in all devices. It is worth noting that the improvement takes place in the whole device as indicated by a uniform shift of the entire $R(T)$ along the $T$ axis. A similar effect has been already reported in Ref. [28] and several possible reasons have been put forward such as degassing or thermally induced stress releasing.

Further increasing the $I_{\mathrm{EA}}$ leads to the development of a second step in the $R(T)$ curve corresponding to the local modification of the critical temperature $T_{c}^{\prime}$ of the weak link [see region II in Figs. 4(b) and 4(c)]. The 
TABLE II. Fitting parameters for the $I_{c}(B)$ oscillations of the nanoSQUID after EA14. The parameters definitions are the same as in Table I.

\begin{tabular}{lccccccc}
\hline \hline$T(\mathrm{~K})$ & $I_{c 1}(\mu \mathrm{A})$ & $\varphi_{c 1}(\mathrm{rad})$ & $I_{c 2}(\mu \mathrm{A})$ & $\varphi_{c 2}(\mathrm{rad})$ & $\alpha_{I}$ & $L_{K 1}(\mathrm{pH})$ & $L_{K 2}(\mathrm{pH})$ \\
\hline 1.8 & $41 \pm 5$ & $5 \pm 1$ & $27 \pm 5$ & $6 \pm 1$ & $0.2 \pm 0.1$ & $40 \pm 9$ & $73 \pm 18$ \\
3 & $42 \pm 5$ & $5 \pm 1$ & $24 \pm 5$ & $5 \pm 1$ & $0.3 \pm 0.1$ & $39 \pm 9$ & $68 \pm 20$ \\
5 & $25 \pm 3$ & $4 \pm 1$ & $16 \pm 3$ & $4 \pm 1$ & $0.2 \pm 0.1$ & $53 \pm 15$ & $82 \pm 26$ \\
\hline \hline
\end{tabular}

observed deterioration in region II could be attributed to the high current density, which deforms the constriction and induces local oxidation or alloying with the substrate. Adopting a criterion of $0.1 \Omega$ to determine $T_{c}^{\prime}$, it is possible to track its evolution. The most striking result is a sudden improvement of the critical temperature [see region III in Figs. 4(b) and 4(c)] accompanied by a sharp decrease of the normal-state resistance $R_{10 \mathrm{~K}}$ for EA12 and EA13 [Fig. 4(c)]. This behavior is carefully confirmed for samples S1 and S4 (see Supplemental Material [29]). We speculate that in this region, the Joule heating produced by the high current densities induces grain coalescence, grain growth, and improved crystallinity. This in turn reduces the resistivity and enhances the mechanical properties (stress relaxation). As a consequence of this annealing process, the critical current of the device decreases since the density of defects acting as pinning centers, decreases. Similar phenomena have been reported in $\mathrm{Mo}_{6} \mathrm{~S}_{3} \mathrm{I}_{6}$ nanowires [33], Si microwires [34], and $\mathrm{Cu}$ microwires [35], and has been modeled numerically for the case of Pt nanowires [36]. What is particularly interesting in the present study is that rather minor changes in the mechanical and structural properties of $\mathrm{Nb}$, have a sizable impact on its superconducting properties. Additional local material properties investigation (such as transmission electron microscopy or atom probe tomography [37]) are needed to confirm this interpretation. Eventually, further electroannealing steps lead to a clear deterioration of the sample (see also sample S4 in the Supplemental Material [29]).

\section{Critical current versus magnetic field oscillations after electroannealing}

Let us now analyse the SQUID critical current oscillations $I_{c}(B)$ when rendering the SQUID in different states using the EA process. Figure 5 shows the $I_{c}(B)$ curves obtained after the last EA step (EA14) at three different temperatures together with the fits using the model described in Sec. III B. The fitting parameters are shown in Table II. After EA14, the asymmetry in critical currents increases with temperature and is comparable to the values obtained for the pristine nanoSQUID. In contrast to that, the critical phase differences are considerably smaller than those observed in the pristine SQUID, indicating a significant decrease of the effective length of the weak links [26]. Moreover, the critical phase differences for both
Dayem-bridge arms are almost identical, which is consistent with the observation that the $I_{c}(B)$ curves are centered around zero field as expected from Eq. (3). Given the decrease in $d_{\text {eff }}$ and that $L_{K}(T)=\mu_{0} \lambda(T)^{2} d_{\text {eff }} / A$, the significant increase in kinetic inductance implies, as expected, a reduced cross section of the constriction and a diminished density of superconducting carriers. It is worth noting that additional experiments are undertaken on sample S4 to monitor the evolution of the coherence length over the EA steps (see Supplemental Material [29]), which show minor changes in $\xi$.

By comparing Fig. 2 with Fig. 5, we observe that the global effect of EA is to significantly reduce the critical current and its oscillation amplitude with respect to magnetic field. Figure 6 shows the evolution of the maximum value of the critical current $I_{c}^{\max }$ along with the relative peak-to-peak modulation amplitude $\Delta I_{c} / I_{c}^{\max }$ during successive EA steps for $T=5 \mathrm{~K}$. We observe that during the first four EA steps the critical current of the SQUID exhibits a $2.5 \%$ rise concomitant with the slight resistance decrease and $T_{C}^{\prime}$ increase shown in Fig. 4. From the sixth EA step the critical current displays a significant global reduction until reaching EA08 where both the critical current and its oscillations disappear. Thereafter, the critical current is restored to a fraction of the initial value for EA12 to EA14 while the relative peak-to-peak modulation amplitude remains zero until before the last EA step. The fact that at EA13 the critical current has been

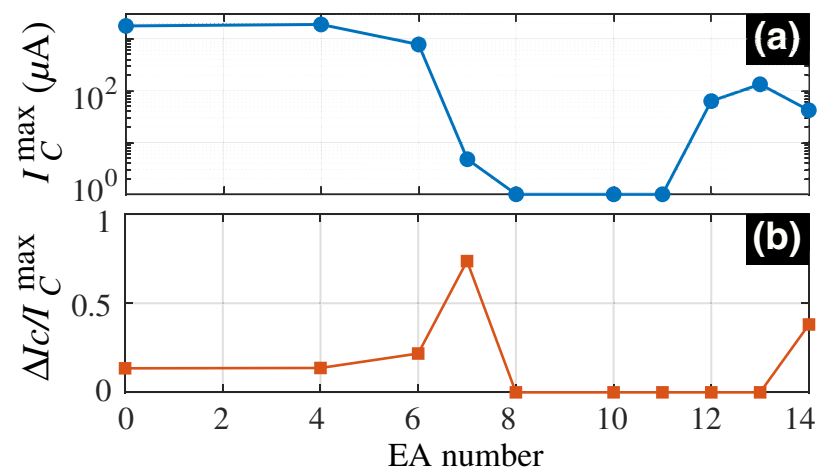

FIG. 6. Maximum value of the critical current $I_{c}^{\max }$ (a) along with the relative peak-to-peak amplitude oscillation $\Delta I_{c} / I_{c}^{\max }$ (b), after each EA step for $T=5 \mathrm{~K}$ (sample S1). EA numbers with no data point correspond to situations with no measurements. 
substantially recovered without however exhibiting critical current oscillations, suggests that only one junction has resurrected.

\section{Thermal regimes and retrapping}

As shown in Fig. 1(c) the pristine $\mathrm{Nb}$ nanoSQUIDs exhibit strongly hysteretic current-voltage characteristics. This is an unwanted feature limiting their flux resolution and operation speed. Unlike SQUIDs based on tunnelbarrier Josephson junctions, where the hysteresis results from the junction capacitance, in Dayem bridges the hysteresis arises from Joule heating. Figure 7(a) shows the temperature dependence of the critical current $I_{c}(T)$ obtained by sweeping up the applied current, together with the retrapping current $I_{r}(T)$ obtained by sweeping down the applied current. There is a narrow temperature region $T_{h}<T<T_{c}$ where the $V-I$ characteristic is nonhysteretic and the nanoSQUID can be operated in dissipative mode. By performing successive EA processes, it is possible to enhance the relative temperature range $1-T_{h} / T_{c}^{\prime}$ of nonhysteretic response, as shown by the blue colored area in the inset of Fig. 7(a). This enlargement of the nonhysteretic regime comes at the expense of reducing the critical current of the device. For the sake of illustration, Fig. 7(b)-(d) shows the evolution of $I_{c}(T)$ and $I_{r}(T)$ at different EA steps (main panels) along with the SQUID oscillation at the indicated temperatures. Remarkably, no compromise

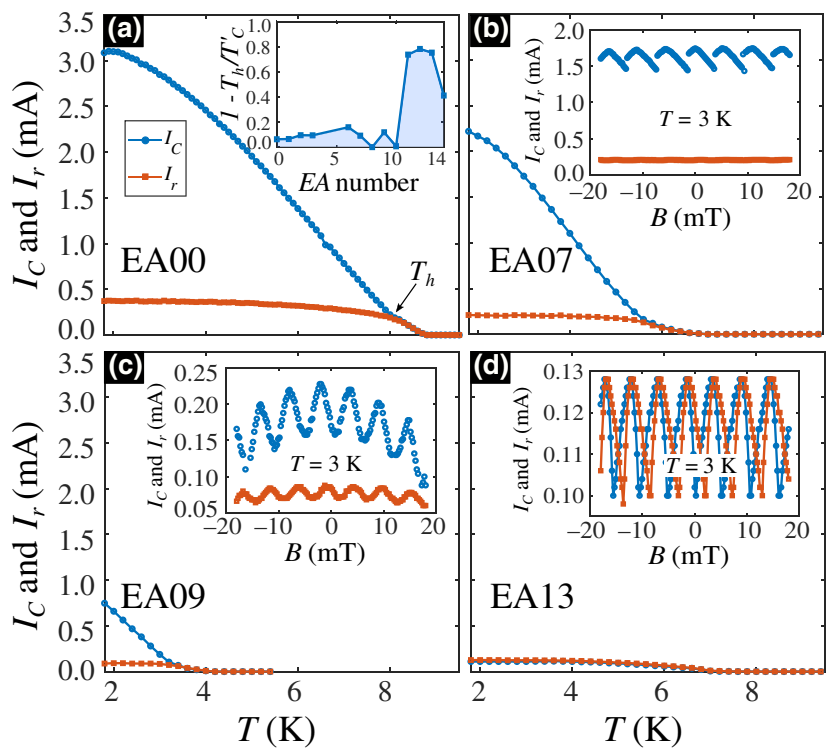

FIG. 7. Critical current $I_{c}$ and retrapping current $I_{r}$ of sample S4 as a function of temperature for the pristine sample (a), after EA07 (b), EA09 (c), and EA13 (d). The temperature above which the current-voltage characteristics become reversible, $T_{h}$, is indicated in (a). The blue colored area in the inset of (a) shows that the range where the SQUID can be operated in the dissipative state is increased by EA process at expense of reducing $I_{c}$. The insets in (b)-(d) show $I_{c}(B)$ and $I_{r}(B)$ for the corresponding EA step at $3 \mathrm{~K}$.
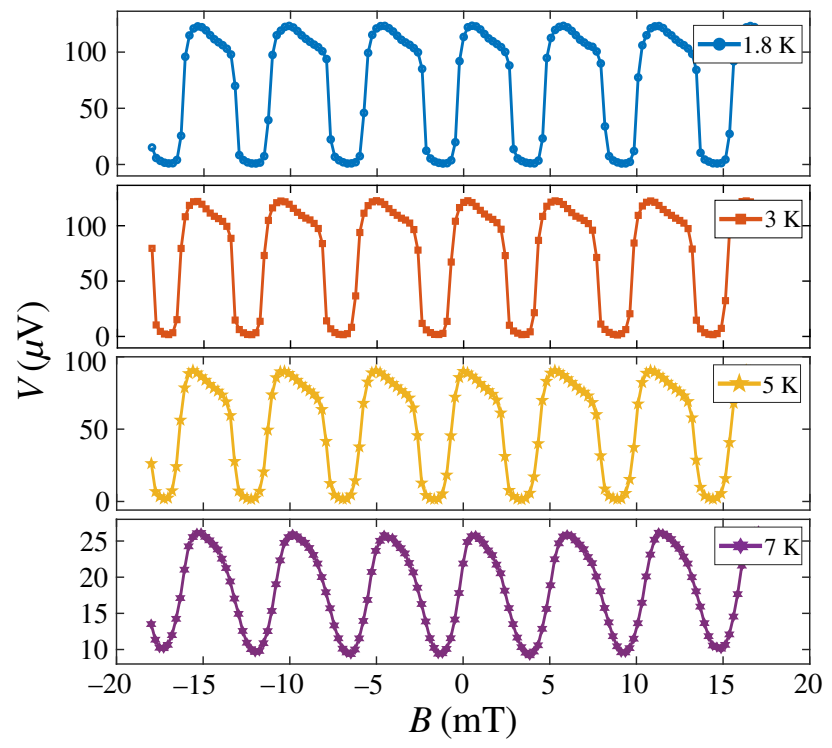

FIG. 8. Voltage oscillations observed for sample S4 after EA13. The bias currents are 132, 130, 104, and $30 \mu \mathrm{A}$ for $T=1.8,3,5$, and $7 \mathrm{~K}$, respectively.

is made on the critical temperature of the weak link $T_{c}^{\prime}$, which, in contrast, is largely increased by the EA process compared to intermediate EA steps, as shown in Fig. 4(c). This is a truly striking and unique property of the electroannealed nanoSQUIDs. Note that SQUID oscillations are absent for the retrapping current in Fig. 7(b) indicating that the local temperature at the weak link is above $T_{c}^{\prime}$ due to the formation of a hot spot. However, clear SQUID oscillations of the retrapping current after EA09 indicate that the local temperature at the weak link does not exceed $T_{c}^{\prime}$ [11,38]. Recently, a time-dependent thermal model for a weak link made of a superconducting constriction was proposed by Gupta et al. [39]. The analysis of the $I_{r}(T)$ for our $\mathrm{Nb}$ nanoSQUIDS based on this model is discussed in the Supplemental Material [29].

Practical SQUIDs are operated in the dissipative state as a magnetic field sensor, where the device produces an oscillating output voltage as a function of the applied magnetic flux. In the early stages of EA, the fully resistive state generated by the high critical current and the hot-spot formation prevent the device presenting voltage oscillations. As a result of the EA process, the decrease of the critical current allows the SQUID interference to persist in the voltage state. Figure 8 shows the voltage oscillations obtained after EA13. This result illustrates the potential of the electroannealing protocol to improve the properties of a SQUID and to switch from a hysteretic behavior to a nonhysteretic nanoSQUID.

\section{CONCLUSION}

In summary, we demonstrate the fabrication and in situ tuning of a nanoSQUID, by means of electroannealing. We 
show that the EA process can be useful in a threefold manner: (i) to improve the superconducting properties (higher $T_{c}^{\prime}, I_{c}$, and lower normal resistance) in the Josephson junctions for weak annealing currents, (ii) to suppress these superconducting properties of the weak links by intermediated applied currents, and (iii) to restore the superconducting properties with larger currents except for $I_{c}$, which remain substantially lower than the value corresponding to the pristine state. We measure oscillations in the critical current as a function of magnetic field and demonstrate that the oscillation amplitude and asymmetry can be tuned over a broad range by successive electroannealing processes. The overall tendency of EA to decrease the critical current makes it possible to modify the thermal behavior of a sample so as to reach a state with nonhysteretic current-voltage characteristics, while substantially preserving the critical temperature of the junctions $T_{c}^{\prime}$. This property makes the EA technique particularly interesting and promising permitting easy adjustment and improvement of the properties of $\mathrm{Nb}$ SQUID sensors. We conclude that the proposed method is able to introduce modification and control of weak links at the nanoscale, with the possibility of adjusting its properties in situ, thus beyond the available state of the art, where weak links are either preformed and not tunable, or resulting from a rather involved nanofabrication procedure and further complex external control. The electroannealing process should be applicable to refractory materials, which can stand substantial heat, such as $\mathrm{NbN}$ and $\mathrm{NbTi}$.

\section{ACKNOWLEDGMENT}

This work is supported by the Fonds de la Recherche Scientifique - FNRS, the Research Foundation - Flanders (FWO) through Grant No. G0A0619N, the Deutsche Forschungsgemeinschaft (DFG) via KO 1303/13-2, and by COST (European Cooperation in Science and Technology) [45] through COST Action CA16218 "NanoCoHybri." The work of A.V.S. is partially supported by CDR J.0151.19 and EQP U.N027.18 of the F.R.S.-FNRS. The authors thank the ULiège Microscopy facility CAREM for part of the SEM investigations.

[1] J. Clarke and A. I. Braginski, The SQUID Handbook. Vol. I: Fundamentals and Technology of SQUIDs and SQUID Systems (Wiley-VCH, 2004).

[2] J. Clarke and A. I. Braginski, The SQUID Handbook. Vol. II: Applications of SQUIDs and SQUID Systems (WILEYVCH Verlag GmbH \& Co. KGaA, Weinheim, 2006).

[3] C. Granata and A. Vettoliere, Nano superconducting quantum interference device: A powerful tool for nanoscale investigations, Phys. Rep. 614, 1 (2016).

[4] D. D. Awschalom, J. R. Rozen, M. B. Ketchen, W. J. Gallagher, A. W. Kleinsasser, R. L. Sandstrom, and B. Bumble, Low-noise modular microsusceptometer using nearly quantum limited dc SQUIDs, Appl. Phys. Lett. 53, 2108 (1988).

[5] M. Hatridge, R. Vijay, D. H. Slichter, J. Clarke, and I. Siddiqi, Dispersive magnetometry with a quantum limited SQUID parametric amplifier, Phys. Rev. B 83, 134501 (2011).

[6] M. J. Martínez-Pérez and D. Koelle, NanoSQUIDs: Basics \& recent advances, Phys. Sci. Rev. 2, 20175001 (2017).

[7] K. K. Likharev, Superconducting weak links, Rev. Mod. Phys. 51, 101 (1979).

[8] A. A. Golubov, M. Y. Kupriyanov, and E. Il'ichev, The current-phase relation in Josephson junctions, Rev. Mod. Phys. 76, 411 (2004).

[9] D. Hazra, J. R. Kirtley, and K. Hasselbach, Nanosuperconducting quantum interference devices with suspended junctions, Appl. Phys. Lett. 104, 152603 (2014).

[10] K. Hasselbach, D. Mailly, and J. R. Kirtley, Microsuperconducting quantum interference device characteristics, J. Appl. Phys. 91, 4432 (2002).

[11] S. Biswas, C. B. Winkelmann, H. Courtois, and A. K. Gupta, Josephson coupling in the dissipative state of a thermally hysteretic $\mu$-SQUID, Phys. Rev. B 98, 174514 (2018).

[12] J. J. A. Baselmans, B. J. van Wees, and T. M. Klapwijk, Direct demonstration of circulating currents in a controllable $\pi$-SQUID generated by a 0 to $\pi$ transition of the weak links, Phys. Rev. B 65, 224513 (2002).

[13] T. Schäpers, J. Malindretos, K. Neurohr, S. Lachenmann, A. van der Hart, G. Crecelius, H. Hardtdegen, H. Lüth, and A. A. Golubov, Demonstration of a current-controlled threeterminal $\mathrm{Nb}-\mathrm{In}_{x} \mathrm{Ga}_{1-x} \mathrm{As} / \mathrm{InP}$ Josephson contact, Appl. Phys. Lett. 73, 2348 (1998).

[14] G. J. Podd, G. D. Hutchinson, D. A. Williams, and D. G. Hasko, Micro-SQUIDs with controllable asymmetry via hot-phonon controlled junctions, Phys. Rev. B 75, 134501 (2007).

[15] R. Winik, I. Holzman, E. G. Dalla Torre, E. Buks, and Y. Ivry, Local tuning of the order parameter in superconducting weak links: A zero-inductance nanodevice, Appl. Phys. Lett. 112, 122601 (2018).

[16] A. Finkler, Y. Segev, Y. Myasoedov, M. L. Rappaport, L. Ne'eman, D. Vasyukov, E. Zeldov, M. E. Huber, J. Martin, and A. Yacoby, Self-aligned nanoscale SQUID on a tip, Nano Lett. 10, 1046 (2010).

[17] V. S. Zharinov, X. D. A. Baumans, A. V. Silhanek, E. Janssens, and J. Van de Vondel, Controlled electromigration protocol revised, Rev. Sci. Instrum. 89, 043904 (2018).

[18] X. D. A. Baumans, Ph.D. thesis, Université de Liège, Belgium, 2018. https://orbi.uliege.be/handle/2268/228346

[19] W. Keijers, X. D. A. Baumans, R. Panghotra, J. Lombardo, V. S. Zharinov, Roman B. G. Kramer, A. V. Silhanek, and J. Van de Vondel, Nano-SQUIDs with controllable weak links created via current-induced atom migration, Nanoscale 10, 21475 (2018).

[20] A. F. Mayadas, R. B. Laibowitz, and J. J. Cuomo, Electrical characteristics of rf-sputtered single-crystal niobium films, J. Appl. Phys. 43, 1287 (1972).

[21] M. Tinkham, Introduction to Superconductivity (Dover Publications, Inc., McGraw-Hill, New York, 1996), 2nd ed. 
[22] P. H. Kes and C. C. Tsuei, Two-dimensional collective flux pinning, defects, and structural relaxation in amorphous superconducting films, Phys. Rev. B 28, 5126 (1983).

[23] M. M. Khapaev, M. Y. Kupriyanov, E. Goldobin, and M. Siegel, Current distribution simulation for superconducting multi-layered structures, Supercond. Sci. Technol. 16, 24 (2003).

[24] D. Drung, Introduction to Nb-Based SQUID sensors, IEEE/CSC ESAS Superconductivity News Forum (2016).

[25] D. Hazra, Nanobridge superconducting quantum interference devices: Beyond the Josephson limit, Phys. Rev. B 99, 144505 (2019).

[26] A. Murphy and A. Bezryadin, Asymmetric nanowire SQUID: Linear current-phase relation, stochastic switching, and symmetries, Phys. Rev. B 96, 094507 (2017).

[27] J. Jaycox and M. Ketchen, Planar coupling scheme for ultra low noise de squids, IEEE Trans. Magn. 17, 400 (1981).

[28] J. Lombardo, Z. L. Jelić, X. D. A. Baumans, J. E. Scheerder, J. P. Nacenta, V. V. Moshchalkov, J. Van de Vondel, R. B. G. Kramer, M. V. Milošević, and A. V. Silhanek, In situ tailoring of superconducting junctions via electro-annealing, Nanoscale 10, 1987 (2018).

[29] See Supplemental Material at http://link.aps.org/supple mental/10.1103/PhysRevApplied.15.034016 for additional SEM, AFM and weak-link characterization measurements, the evolution of the coherence length during EA steps, the description of finite-element-method simulation [40,41] and the application of a dynamic thermal model [11,39,4244].

[30] W. DeSorbo, Effect of dissolved gases on some superconducting properties of niobium, Phys. Rev. 132, 107 (1963).

[31] C. C. Koch, J. O. Scarbrough, and D. M. Kroeger, Effects of interstitial oxygen on the superconductivity of niobium, Phys. Rev. B 9, 888 (1974).

[32] J. Halbritter, On the oxidation and on the superconductivity of niobium, Appl. Phys. A 43, 1 (1987).

[33] M. Hummelgård, R. Zhang, T. Carlberg, D. Vengust, D. Dvorsek, D. Mihailovic, and H. Olin, Nanowire transformation and annealing by Joule heating, Nanotechnology 21, 165704 (2010).

[34] G. Bakan, N. Khan, A. Cywar, K. Cil, M. Akbulut, A. Gokirmak, and H. Silva, Self-heating of silicon microwires: Crystallization and thermoelectric effects, J. Mater. Res. 26, 1061 (2011).
[35] M. Tohmyoh and H. Ishihara, Local modification of $\mathrm{Cu}$ microwires by Joule heating, Appl. Phys. Express 6, 077302 (2013).

[36] K. S. McGarrity, B. Gao, and J. M. Thijssen, Simulation of Joule annealing in nanoscale Pt wires, Comput. Mater. Sci. 50, 3043 (2011).

[37] Y. J. Kim, R. Tao, R. F. Klie, and D. N. Seidman, Direct atomic-scale imaging of hydrogen and oxygen interstitials in pure niobium using atom-probe tomography and aberration-corrected scanning transmission electron microscopy, ACS Nano 7, 732 (2013).

[38] N. Kumar, T. Fournier, H. Courtois, C. B. Winkelmann, and A. K. Gupta, Reversibility of Superconducting $\mathrm{Nb}$ Weak Links Driven By The Proximity Effect in a Quantum Interference Device, Phys. Rev. Lett. 114, 157003 (2015).

[39] A. K. Gupta, N.1 Kumar, and S. Biswas, Temperature and phase dynamics in superconducting weak-link, J. Appl. Phys. 116, 173901 (2014).

[40] COMSOL Multiphysics ${ }^{\circledR}$ v. 5.4. COMSOL AB, Stockholm, Sweden.

[41] A. D. Avery, S. J. Mason, D. Bassett, D. Wesenberg, and B. L. Zink, Thermal and electrical conductivity of approximately 100-nm permalloy, $\mathrm{Ni}, \mathrm{Co}, \mathrm{Al}$, and $\mathrm{Cu}$ films and examination of the Wiedemann-Franz law, Phys. Rev. B 92, $21(2015)$

[42] A. V. Gurevich and R. G. Mints, Self-heating in normal metals and superconductors, Rev. Mod. Phys. 59, 941 (1987).

[43] X. D. A. Baumans, V. S. Zharinov, E. Raymenants, S. Blanco Alvarez, J. E. Scheerder, J. Brisbois, D. Massarotti, R. Caruso, F. Tafuri, E. Janssens, V. V. Moshchalkov, J. Van de Vondel, and A. V. Silhanek, Statistics of localized phase slips in tunable width planar point contacts, Sci. Rep. 7, 44569 (2017).

[44] D. Hazra, J. R. Kirthley, and K. Hasselbach, Retrapping Current in Bridge-Type Nano-SQUIDs, Phys. Rev. Appl. 4, 024021 (2015).

[45] www.cost.eu.

Correction: One of the support statements in the Acknowledgment section contained incomplete information and has been fixed. A corresponding reference has also been added. 